\title{
A Hadronic Model of the Fermi Bubbles: Cosmic-Rays in a Galactic Breeze
}

\author{
Gwenael Giacinti* \\ Max-Planck-Institut für Kernphysik, Postfach 103980, 69029 Heidelberg, Germany \\ E-mail: giacinti@mpi-hd.mpg.de
}

\section{Andrew M. Taylor}

Dublin Institute for Advanced Studies, 31 Fitzwilliam Place, Dublin 2, Ireland

E-mail: taylora@cp.dias.ie

\begin{abstract}
We present a self-consistent model of the Fermi Bubbles, described as a decelerating outflow of gas and non-thermal particles produced within the Galactic center region, on a $\sim 100 \mathrm{Myr}$ timescale. Motivated by observations, we use an outflow with velocity of a few hundreds of $\mathrm{km} / \mathrm{s}$, which is slower than the velocities used in models describing the Bubbles as a more recent outburst. We take into account cosmic-ray (CR) energy losses due to proton-proton interactions, and calculate the resulting gamma-ray emission. Our model can reproduce both the spatial morphology and the spectra of the Bubbles, on a range of different scales. We find that CR diffusing and advecting within a breeze outflow result in an approximately flat surface brightness profile of the gamma-ray emission, as observed by Fermi satellite. Finally, we apply similar outflow profiles to larger Galactocentric radii, and investigate their effects on the CR spectrum and boron-to-carbon ratio. Hardenings can appear in the spectrum, even in cases with equal CR diffusion coefficients in the disk and halo. It is postulated that this hardening effect may relate to the observed hardening feature in the CR spectrum at a rigidity of $\sim 200 \mathrm{GV}$.
\end{abstract}

35th International Cosmic Ray Conference - ICRC2017

10-20 July, 2017

Bexco, Busan, Korea

${ }^{*}$ Speaker. 


\section{Introduction}

A number of indications that the center of our Galaxy feeds a wind has been found over the last few decades. This body of evidence has been provided from observations in a broad energy range: radio HI [1], infrared (IR) [2], and X-rays [3]. IR observations have also indicated that this wind continues further away [4], and that it may be responsible for the larger structures observed out of the Galactic plane. Absorption line features in the spectra of Active Galactic Nuclei (AGN) can be used as a probe of the structure of the gas flow: See [5], whose results indicate the presence of a coherent gas flow, consistent with an outflow being directed away from the Galactic plane. More recent gamma-ray and radio observations have shown the presence of extended non-thermal particle populations in bubble-like structures in the halo, both above and below the Galactic center (GC), see $[6,7,8,9]$. The current picture seems to indicate that cosmic-rays (CR) and hot gas are conveyed out from the GC region into the halo within a Galactocentric outflow. As for the velocity of this outflow, values of $\sim 300 \mathrm{~km} / \mathrm{s}$ have been suggested in the region close to the Galactic disk (within $\sim$ a couple of kiloparsecs), from the weakness of the X-ray features associated with the edge of the bubbles $[6,10,11,12]$. At distances $\sim 4 \mathrm{kpc}$ and $\sim 9 \mathrm{kpc}$ away of the Galactic plane, observations of high velocity clouds suggest velocities of about $\sim 150 \mathrm{~km} / \mathrm{s}$, cf. [5]. Further out, towards the edges, velocities are $\lesssim 100 \mathrm{~km} / \mathrm{s}$. In radio [9], the bending observed in the outflow at high latitudes may be related to the motion of our Galaxy towards Andromeda.

In the present work, we study the secondary signatures produced by CR embedded in outflows. See also [13] for more details. In Section 2, we focus on the Fermi bubbles. We then apply, in Section 3, a similar outflow velocity profile at larger Galactocentric radii, and investigate the possible traces it would leave on local CR observables, should such an outflow exist locally. We present our conclusions in Section 4.

\section{CR and $\gamma$-rays associated with a Galactocentric Outflow}

We describe CR propagation within an outflow from the GC using the diffusion-advection equation:

$$
\frac{\partial \psi_{\mathrm{CR}}}{\partial t}=\nabla \cdot\left(D \nabla \psi_{\mathrm{CR}}-V \psi_{\mathrm{CR}}\right)+\frac{\partial}{\partial p}\left[\frac{p}{3}(\nabla \cdot V) \psi_{\mathrm{CR}}\right]-\frac{\psi_{\mathrm{CR}}}{\tau_{\mathrm{CR}}}+Q_{\mathrm{CR}}
$$

where $\psi_{\mathrm{CR}}(\mathbf{r}, p, t)$ denotes the CR density per unit of particle momentum $p$ at $\mathbf{r}$, and $Q_{\mathrm{CR}}$ is the source term. A diffusion scattering length scale of $\lambda_{10 \mathrm{GV}}=3 D_{10 \mathrm{GV}} / c=0.3 \mathrm{pc}$ is adopted. For CR protons, $\tau_{\mathrm{CR}}=\tau_{p p}$ is the energy loss time scale from $p p$ interactions, while for CR nuclei, $\tau_{\mathrm{CR}}$ is the interaction time scale. We adopt a divergence free outflow velocity profile, whose $z$-dependence (in a cylindrical coordinate system where the $z$-axis is perpendicular to the disk) takes the form

$$
V \cdot \hat{\mathbf{z}}=v_{\max } e^{\frac{1}{2}\left(1-\frac{d}{z}\right)} \times \frac{2}{1+z / d},
$$

with $v_{\max }=300 \mathrm{~km} \mathrm{~s}^{-1}$ and $d=1 \mathrm{kpc}$. Such an outflow profile is motivated by the observations discussed above. For such a profile, a timescale of $\mathscr{O}(100 \mathrm{Myr})$ is required in order for the outflow to fill a region beyond the bubbles. As for the source of this outflow, both a past AGN outburst 

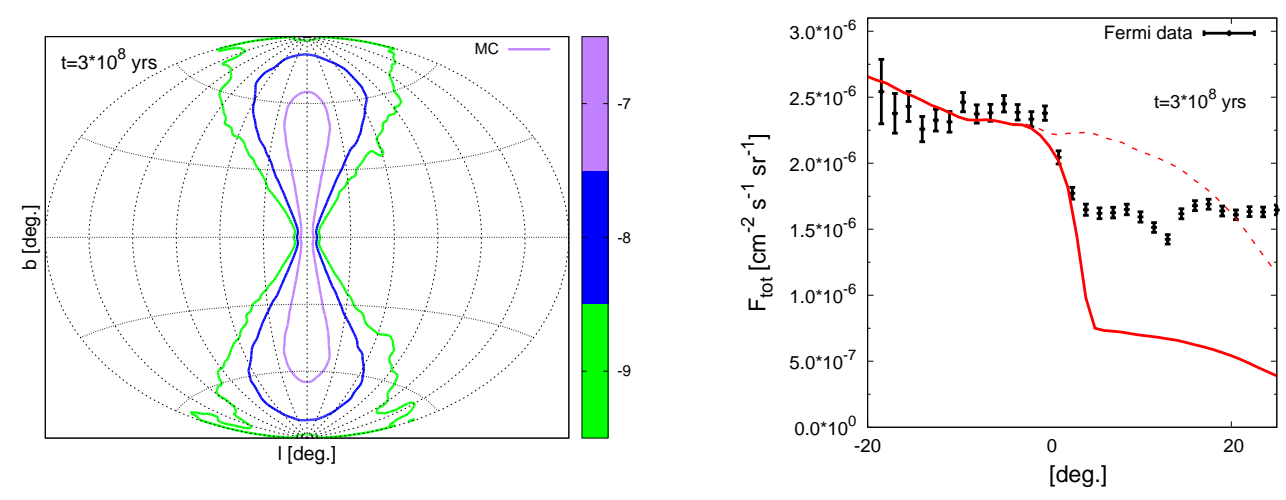

Figure 1: Left: Contour plots showing $\log _{10}$ of the $\gamma$-ray flux surface brightness $\left(\mathrm{cm}^{-2} \mathrm{~s}^{-1} \mathrm{sr}^{-1}\right)$ from the bubbles following the interaction of $\mathrm{CR}$ in the outflow with the gas present. The different line colours indicate the corresponding contour value, whose values are provided in the colour bar in the side-panel. Right: A comparison of the edge of the $1-2 \mathrm{GeV} \gamma$-ray bubble from our outflow model with that from the Fermi observation analysis. The angle $\vartheta$ is counted from the edge of the bubble. It is noted that for the energy bin considered, at large $\vartheta$, further diffuse $\gamma$-ray background [18] dominates the observed flux, with the model values sitting below this level in this region. The solid line result adopts a decrease in the gas density at the bubble edge whereas the dashed line result assumes a constant density throughout.

event, and a starburst phase or a sustained outflow driven by star formation in the Galactic centre have been proposed in the literature. Ref. [14] claims that the present velocity data are not conclusive on the type of source responsible for this outflow. In the following, we then keep the discussion general, adopting instead the specific velocity profile of Eq. (2.2) as the starting point in our calculations. Such a profile broadly encapsulates the velocity profile of a "breeze" solution for the isothermal outflow problem $[15,16]$. For such a solution, the wind is launched sufficiently subsonically that it accelerates without becoming transonic, before decelerating after the Bondi radius. The corresponding gas density profile of our breeze description plateaus within the decelerating flow phase. This motivates our naive constant density description for gas in the halo.

We utilise a Monte Carlo approach to solve (2.1). We assume that our source term, $Q_{C R}$, is constant in time and located at the GC region. The copresence of the resultant accumulated $\mathrm{CR}$ with ambient gas gives rise to $\gamma$-ray bubble emission through $\pi^{0}$ production generated in $p p$ interactions. This emission may potentially account for the observed $\gamma$-rays from the bubbles. To calculate this emission, the CR density throughout the outflow region is convolved with the target gas density in the outflow. As motivated on theoretical (e.g. [17]) and observational (e.g. [11]) grounds, we adopt a constant gas density within the Bubbles at the level $3 \times 10^{-3} \mathrm{~cm}^{-3}$. A $\gamma$-ray density map and a comparison of the $\gamma$-ray bubble-edge profile with Fermi data are shown in Fig. 1. For these results, a CR luminosity of $10^{40} \mathrm{erg} \mathrm{s}^{-1}$ has been adopted for the source at the GC.

As can be seen from Fig. 1, a flat surface brightness profile for the bubbles is obtained following the assumption that the velocity profile of the bubbles is described by Eq. (2.2). We note though that in reality a range of velocity profiles can provide such a uniform brightness. See for example [19]. In general, we find that for the case of a constant density ambient medium description, the present $\gamma$-ray data can be said to prefer decelerating profiles. Instead, for decreasing gas density profiles, a sharper fall-off in the velocity profile, than that adopted in Eq. (2.2), would be required. Although the cutoff at the bubble edges is not well described by the simple constant 
density gas model (see dashed line in Fig. 1), a steeper cutoff in the $\gamma$-ray profile can be achieved by a sudden change in the density of the gas at the bubble edge (see solid line), as motivated in certain models [20]. A further motivation for such an origin for the bubble edges comes from a comparison of their morphology as seen in $\gamma$-rays [6] and in radio [9]. If $\mathrm{GeV}$ protons and electrons respectively give rise to the $\gamma$-ray and radio emissions, it would be curious that the electrons extend out to larger latitudes than the protons. Such a difference between $\gamma$-ray and radio data morphologies disfavours simple leptonic scenarios for the $\gamma$-ray bubbles. Despite such challenges, however, more involved diffuse acceleration models supporting a scenario in which both the radio and $\gamma$-ray emission are leptonic in origin are also presently viable [21]. One simple explanation for the difference in latitudinal profiles in the radio and $\gamma$-ray emission is that both protons and electrons possess extended distributions, and that the difference in morphology of the secondary emission they produce is dictated by differing distributions of target gas and magnetic fields.
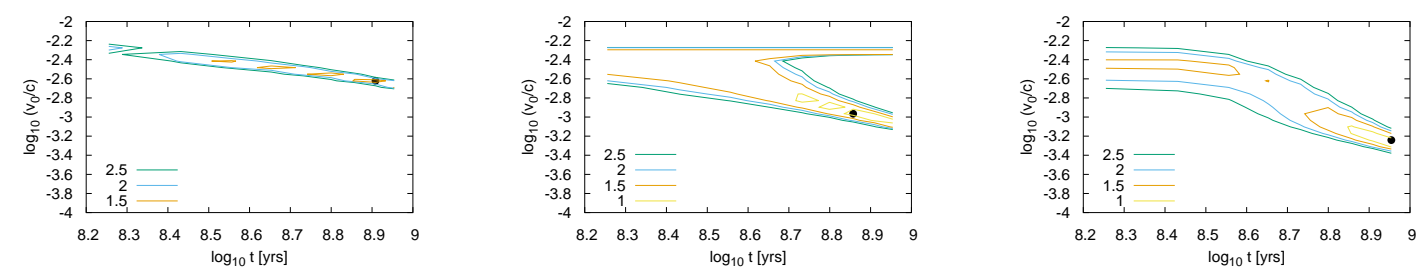

Figure 2: Plots showing $\chi_{\text {d.o.f. }}^{2}$ contours for fits to the $\gamma$-ray flux surface brightness profile of the Fermibubbles using Fermi satellite measurements in the range $\vartheta<2$ [deg.]. The different contour plots cover a range of different cases for the distance $d$, over the range: $d=0.3 \mathrm{kpc}$ (left); $d=1 \mathrm{kpc}$ (centre); $d=2 \mathrm{kpc}$ (right). In each plot, the position of the best-fit parameters is marked with a black circle.

With regards the parameter $d$, which dictates the turnover distance in the outflow velocity profile described by Eq. (2.2), a comparison of the fits to the radial gamma-ray profile of the Fermi bubbles is provided in Fig. 2, through a consideration of the $\chi_{\text {d.o.f. }}^{2}$ contours. The left panel in this figure shows that for the majority of the parameter space, small values of $d$ are problematic, with the large $\chi_{\text {d.o.f. }}^{2}$ values obtained reflecting the fact that such values lead to centrally brightened profiles, incompatible with the flat profile suggested by the data. However, the centre and right panels show that the results for such intermediate and "large" values of $d$ both show considerable regions of parameter space able to provide sufficiently flat profiles in agreement with that measured.

\section{Local Outflow and CR Fluxes at Earth}

We now study the impact on CR observables of a local outflow, whose velocity gradient becomes negative above a given height $d$ in the halo. Such velocity profiles may be motivated in some models (see e.g. the simulations of [22]).

As a first approximation, we ignore here any variation of CR sources or propagation parameters in the radial direction from the GC axis. We assume that a one dimensional model is able to encapsulate CR propagation in the halo. Numerically solving the planar diffusion-advection equation in $z$ and $E$, for any arbitrary profiles of $V(z)$ and $D(E)$, we investigate the effect of the 

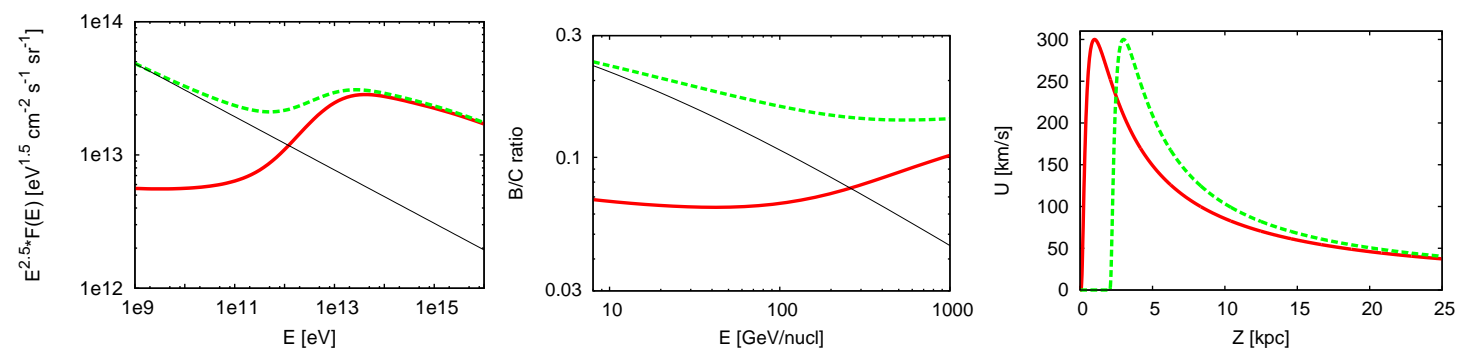

Figure 3: $\mathrm{CR}$ flux (left panel) and $\mathrm{B} / \mathrm{C}$ ratio (centre) at $z=0$, for the outflow profiles displayed in the right panel. $D_{10 \mathrm{GV}}=3 \times 10^{28} \mathrm{~cm}^{2} \mathrm{~s}^{-1}, \delta=1 / 3, H=25 \mathrm{kpc}, n(z)=0.85 \mathrm{~cm}^{-3}$ for $|z| \leq h$ and $10^{-3} \mathrm{~cm}^{-3}$ otherwise, $\mathrm{CR}$ spectrum at sources $\propto E^{-2.37}$, and total power injected in CR at $|z| \leq h$ set to $\approx 3.3 \times 10^{39} \mathrm{erg} \mathrm{pc}^{-2} \mathrm{yr}^{-1}$. Each scenario is represented by the same line type on each panel. Thin black line for the "best fit" model of [25] with $V=0$.

advection velocity profile on CR observables. We verified that our code accurately reproduces the expected CR density profiles in the halo for the known cases of $V=\operatorname{cst}[23]$ and $V \propto z$ [24], which are constant and decreasing with $z$, respectively. On the contrary, the $V(z)$ profiles we consider below lead to an increase of CR density above $d$, decreasing again as $z \rightarrow H$ (escape), where $H$ denotes the size of the escape boundary. Physically, the existence of $H$ may correspond to the height at which the magnetic field becomes too weak to confine CR through diffusion. We set $\psi=0$ at $z=H$ as a boundary condition. We determine the steady-state distributions for $\psi_{\mathrm{CR}}(z, E)$, for protons, $\mathrm{B}$ and $\mathrm{C}$ nuclei. For the primary source term, we adopt the prescription: $Q_{\mathrm{A}}=0$ in the halo $(|z|>h=200 \mathrm{pc})$, and $Q_{\mathrm{A}}=f_{\mathrm{A}} Q_{\mathrm{CR}}$ in the disk $(|z| \leq h)$, where $f_{\mathrm{A}}$ is the fraction of species $A$ emitted at the source. For the gas density, we adopt: $n=0.85 \mathrm{~cm}^{-3}$ at $|z| \leq h$, and $n \sim 10^{-3} \mathrm{~cm}^{-3}$ at $|z|>h$. We assume that there are no sources of primary boron. We take $H=25 \mathrm{kpc}$, and express $D$ as $D=D_{10 \mathrm{GV}}(E /(Z \times 10 \mathrm{GV}))^{\delta}$, setting $\delta=1 / 3$ and keeping the same normalization $D_{10 \mathrm{GV}}$ as above. Our code reproduces the B/C expected theoretically both for the "no wind", and "constant wind" cases. In the latter case, the key parameter is $z_{*}=D / V$, which separates out the distances at which diffusion and advection dominate the particle transport. For low CR energies, $z_{*}<H$ and particles advect to the boundary. The $\mathrm{B} / \mathrm{C}$ ratio is constant at such low energies because $z_{*} \propto E^{\delta}$. At higher energies, diffusion to the boundary dominates. Since no abrupt flattening is seen at low energies in the $\mathrm{B} / \mathrm{C}$ data, an advection wind speed of less than $\mathscr{O}\left(10 \mathrm{~km} \mathrm{~s}^{-1}\right)$ is required for $H \sim 10 \mathrm{kpc}$, in the case of $V=$ cst. However, other wind profiles with $V \neq$ cst are not ruled out, such as $V(z) \propto z$. We refer to this scenario as a "Bloemen-like" wind [24]. For such a wind, $z_{*} \propto D^{1 / 2}$, and the spectral slope tends to $-\alpha-\delta / 2$, when $z_{*}<H$. Such a profile, however, would not induce any hardening in the CR spectrum. We show now that hardenings can appear with more complicated wind profiles, and notably with our breeze profile.

In Fig. 3, we calculate the CR spectrum at $z=0$ (left panel), and the $\mathrm{B} / \mathrm{C}$ ratio (centre panel) for $V(z)$ from Eq. (2.2) (red curves), and for a similar profile, namely (2.2) with $z \rightarrow z-2 \mathrm{kpc}$ and $V=0$ at $|z|<2 \mathrm{kpc}$ (green curves). Plots of $V(z)$ are shown in the right panel. For reference, we show with thin black lines the "best fit" of the B/C ratio from [25] for $V=0$. We note that we do not try to fit the data. Instead, we study, caricatural examples in order to explore interesting phenomena allowed by diffusion within a breeze profile, such as the formation of breaks or points 
of inflection. Focusing on the shape of the CR spectrum, one can see a point of inflection in each of the curves. In order to interpret these inflection points, a comparison of the advection and diffusion timescales at different energies must be made. For sufficiently low energy CR, advection wins over diffusion at $z_{*}<z<H$, providing an effective halo height $z_{*}<H$. The outflow then reduces the size of the diffusion "box", within which CR can safely diffuse and return to the observer at $z=0$, from the full size, $H$, down to $\sim z_{*}$. Beyond this distance, $\mathrm{CR}$ do not come back to $z=0$. For higher energy $\mathrm{CR}$, however, the diffusion lengths are considerably larger, allowing diffusion to win over advection in the entire halo, and the problem simplifies to a basic leaky-box of size $H$. With the above parameter values, this happens at energies $E \gtrsim 10^{13-14} \mathrm{eV}$. As can be seen in Fig. 3 (left panel), the CR spectrum then returns to a power-law of the form $\propto E^{-\alpha-\delta=-2.7}$ at such energies. Below $\sim 10^{12-13} \mathrm{eV}$, the CR flux is "suppressed".

A more quantitative description of this behaviour is provided through the consideration of the change of $z_{*}$ with diffusion coefficient, described through the relationship, $z_{*} \propto D^{\beta}$. Here, the typical advection time $t_{\text {adv }}$ does not vary strongly with $z$, on $0.2 \lesssim|z| / \mathrm{kpc} \lesssim 2$. Therefore, for low energy CR $\left(\sim 10^{9-11} \mathrm{eV}\right), \beta \sim 0.5$. The resultant spectrum is then close to that a "Bloemenlike" wind, explaining why the spectral index of the red curve in Fig. 3 (left panel) is harder than 2.7 at such energies. At higher energies, $\beta$ grows larger than 1 and the role of the advection term subsequently quickly turns off. The effective box size abruptly increases from $z_{*} \lesssim$ a few kpc to $H$, and the resultant CR spectrum then becomes harder before softening again and matching the spectrum expected for a standard fixed-size diffusion "box", at $E \gtrsim 10^{13-14} \mathrm{eV}$. Due to this change in box size, the spectrum at high energies is normalised to a larger flux value than the spectrum at low energies.

For the green curve, $V=0$ (i.e. $t_{\mathrm{adv}} \rightarrow \infty$ ) at $|z|<2 \mathrm{kpc}$. In this case, the typical diffusion time equals $t_{\mathrm{adv}}$ at a value $z_{*} \approx 2 \mathrm{kpc}$, for CR with $E \lesssim 10^{11} \mathrm{eV}$. In this energy range, the increase of $z_{*}$ with energy is small compared to $2 \mathrm{kpc}$. For $z$ slightly greater than $2 \mathrm{kpc}$, the advection time decreases quickly with $z$, resulting in $\beta$ being small $(\ll 1)$. The CR spectrum in this low energy region reflects that of the fixed-size diffusion "box" case, with a box size equal to $\approx 2 \mathrm{kpc}$. This is why the spectral index of the green curve in Fig. 3 (left panel) tends to 2.7 at low energies.

For some parameter values, it is possible to make the hardening that we found in the CR spectrum coincide better with the one measured at $200 \mathrm{GV}$ by PAMELA, CREAM and AMS-02. Interestingly, if the high-energy softening is left concealed to higher energies ( $\gtrsim 3 \mathrm{PeV}$ ), one may then explain the $200 \mathrm{GV}$ hardening with the launching of a breeze or wind in the halo, even without invoking a change in $D$ between the disk and the halo. This argument remains valid also for some winds with $d V / d z>0$ at all $z$. These data are most sensitive to the accelerating part of the outflow, while those in Sect. 2 essentially probe the decelerating part of the outflow.

For the same reasons, similar hardenings are expected to appear in the $\mathrm{B} / \mathrm{C}$ ratio at "intermediate" energies, see centre panel. This is not contradictory with present measurements as long as the hardening is left concealed to higher energies or is hidden within the systematics of the present instruments.

\section{Conclusions and perspectives}

We presented in Section 2 a hadronic model of the Fermi bubbles. Assuming that they result 
from a Galactocentric outflow carrying pre-accelerated cosmic-rays, we calculated the gammaray emission produced by the $\mathrm{CR}$ interacting with the gas present in the bubbles. We showed that outflows decelerating with distance to the Galactic disk can reproduce the flat gamma-ray surface brightness of the bubbles, in accordance with the measurements from Fermi satellite. Our description for the outflow profile is enclosed in breeze solutions of isothermal winds. Motivated by the above findings, we studied in Section 3 the impacts that similar types of outflow profiles would have on the CR spectra at Earth, should such outflows exist at larger Galactocentric radii. Competition between CR diffusion and advection in the halo can produce an inflection point in the $\mathrm{CR}$ spectrum at $z=0$. A hardening can appear in the CR spectrum due to the launching of a wind or breeze in the halo, even in the hypothetical, limiting case of equal CR diffusion coefficients in the halo and disk. Although a breeze outflow scenario is currently only motivated for the outflow from the GC region, we conclude from the above results that future observations should be able to test its presence or absence at larger radii, thanks to local CR observables.

\section{Acknowledgments}

AT acknowledges a Schroedinger fellowship at DIAS.

\section{References}

[1] F. J. Lockman, The H I halo in the inner galaxy, Astrophys. J. 283 (1984) 90.

[2] M. Morris, E. Serabyn, The Galactic Center Environment, Annu. Rev. Astron. Astrophys. 34 (1996) 645.

[3] L. X. Cheng, M. Leventhal, D. M. Smith, W. R. Purcell, J. Tueller, A. Connors, D. Dixon, R. L. Kinzer, J. G. Skibo, A Maximum Entropy Map of the 511 keV Positron Annihilation Line Emission Distribution Near the Galactic Center, Astrophys. J. 481 (1997) L43.

[4] J. Bland-Hawthorn, M. Cohen, The Large-Scale Bipolar Wind in the Galactic Center, Astrophys. J. 582 (2003) 246.

[5] B. A. Keeney, C. W. Danforth, J. T. Stocke, S. V. Penton, J. M. Shull, K. R. Sembach, Does the Milky Way Produce a Nuclear Galactic Wind?, Astrophys. J. 646 (2006) 951 [astro-ph/0604323].

[6] M. Su, T. R. Slatyer, D. P. Finkbeiner, Giant Gamma-ray Bubbles from Fermi-LAT: AGN Activity or Bipolar Galactic Wind?, Astrophys. J. 724 (2010) 1044 [arXiv:1005.5480].

[7] R. z. Yang, F. Aharonian, R. Crocker, The Fermi Bubbles Revisited, Astron. Astrophys. 567 (2014) A19 [arXiv:1402.0403].

[8] M. Ackermann et al. [Fermi-LAT Collaboration], The Spectrum and Morphology of the Fermi Bubbles, Astrophys. J. 793 (2014) 64 [arXiv:1407.7905].

[9] E. Carretti, R. M. Crocker, L. Staveley-Smith, M. Haverkorn, C. Purcell, B. M. Gaensler, G. Bernardi, M. J. Kesteven, S. Poppi, Giant Magnetized Outflows from the Centre of the Milky Way, Nature 493 (2013) 66 [arXiv:1301.0512].

[10] J. Kataoka, M. Tahara, T. Totani, Y. Sofue, L. Stawarz, Y. Takahashi, Y. Takeuchi, H. Tsunemi et al., Suzaku observations of the diffuse x-ray emission across the fermi bubbles' edges, Astrophys. J. 779 (2013) 57 [arXiv:1310.3553]. 
[11] T. Fang, X. Jiang, High Resolution X-Ray Spectroscopy of the Local Hot Gas along the 3C 273 Sightline, Astrophys. J. 785 (2014) L24 [arXiv:1403.2028].

[12] A. J. Fox et al., Probing the Fermi Bubbles in Ultraviolet Absorption: A Spectroscopic Signature of the Milky Way's Biconical Nuclear Outflow, Astrophys. J. 799 (2015) L7 [arXiv:1412.1480].

[13] A. M. Taylor, G. Giacinti, Cosmic Rays in a Galactic Breeze, Phys. Rev. D 95 (2017) 023001 [arXiv:1607.08862].

[14] K. C. Sarkar, B. B. Nath, P. Sharma, Supernovae vs. AGN: Clues to the origin of Fermi Bubbles from OVIII/OVII line ratio, arXiv:1610.00719.

[15] J. W. Chamberlain, On the Existence of Slow Solutions in Coronal Hydrodynamics, Astrophys. J. 141 (1965) 320.

[16] E. N. Parker, Dynamical Properties of Stellar Coronas and Stellar Winds, IV. The Separate Existence of Subsonic and Supersonic Solutions, Astrophys. J. 141 (1965) 1463.

[17] R. Feldmann, D. Hooper, N. Y. Gnedin, Circum-Galactic Gas and the Isotropic Gamma Ray Background, Astrophys. J. 763 (2013) 21 [arXiv:1205.0249].

[18] A. A. Abdo et al. [Fermi-LAT Collaboration], The Spectrum of the Isotropic Diffuse Gamma-Ray Emission Derived From First-Year Fermi Large Area Telescope Data, Phys. Rev. Lett. 104, 101101 (2010) [arXiv:1002.3603].

[19] K. C. Sarkar, B. B. Nath, P. Sharma, Multiwavelength features of Fermi bubbles as signatures of a Galactic wind, Mon. Not. R. Astron. Soc. 453 (2015) 3827 [arXiv:1505.03634].

[20] R. M. Crocker, G. V. Bicknell, A. M. Taylor and E. Carretti, A Unified Model of the Fermi Bubbles, Microwave Haze, and Polarized Radio Lobes: Reverse Shocks in the Galactic Center's Giant Outflows, Astrophys. J. 808 (2015) 107 [arXiv:1412.7510].

[21] P. Mertsch, S. Sarkar, Fermi gamma-ray 'bubbles' from stochastic acceleration of electrons, Phys. Rev. Lett. 107 (2011) 091101 [arXiv:1104.3585].

[22] Y. Dubois, R. Teyssier, On the onset of galactic winds in quiescent star forming galaxies, Astron. Astrophys. 477 (2008) 79 [arXiv:0707.3376].

[23] A. J. Owens, J. R. Jokipii, Cosmic rays in a dynamical halo. I - Age and matter traversal distributions and anisotropy for nuclei. II - Electrons, Astrophys. J. 215 (1977) 677.

[24] J. B. G. M. Bloemen, V. A. Dogiel, V. L. Dorman, V. S. Ptuskin, Galactic diffusion and wind models of cosmic-ray transport. I - Insight from CR composition studies and gamma-ray observations, Astron. Astrophys. 267 (1993) 372.

[25] Y. Genolini, A. Putze, P. Salati, P. D. Serpico, Theoretical uncertainties in extracting cosmic-ray diffusion parameters: the boron-to-carbon ratio, Astron. Astrophys. 580 (2015) A9 [arXiv:1504.03134]. 